\title{
Is There a Role for DAT-SPECT Imaging in a Specialty Movement Disorders Practice?
}

\author{
Danny Bega $^{a}$ Paulina Gonzalez-Latapi ${ }^{a}$ Cindy Zadikoff ${ }^{a}$ William Spies ${ }^{b}$ \\ Tanya Simuni ${ }^{\mathrm{a}}$ \\ Departments of a Neurology and ${ }^{b}$ Radiology, Northwestern University Feinberg School of Medicine, Chicago, III., USA
}

\author{
Key Words \\ DaTscan · DAT-SPECT · Parkinson's disease
}

\begin{abstract}
Objective: Analyze indications for ordering DAT-SPECT scans and the clinical impact of scan results on patients evaluated in a movement disorders practice. Background: DATSPECT is FDA approved to evaluate cases of suspected presynaptic dopaminergic deficiency. Little data is available on clinical use and impact of these scans among movement disorders neurologists. Methods: DAT-SPECT scans ordered at the Northwestern University Parkinson's disease (PD) and movement disorders center from 2011-2013 were reviewed. Clinic notes were reviewed for information regarding the indication for ordering each scan, and to assess for any changes in clinical impression or management choices that followed the scan. Results: 83 scans were ordered by four specialists. Scans were commonly ordered to differentiate PD from Essential Tremor $(21.7 \%, n=18)$ or from drug-induced parkinsonism (21.7\%, $n=18)$. In 59\% ( $n=49$ ) of cases, a change in clinical diagnosis or medication regimen occurred within one visit after the scan. The strongest impact was seen for the indication of ET vs. PD in which $72.2 \%(n=13)$ had a change in diagnosis, management, or both. Conclusions: Diagnostic uncertainty in cases of parkinsonism exists even in a tertiary referral center. DAT-SPECT has significant impact on clinical diagnosis and management even in the hands of movement disorders specialists.

(c) 2015 S. Karger AG, Basel
\end{abstract}

\section{Background}

Parkinson's disease (PD) is characterized by degeneration of dopamine-producing neurons in the substantia nigra. The onset of motor symptoms in PD occurs only after approximately $80 \%$ of striatal dopamine and $50 \%$ of nigral dopaminergic cells are lost [1]. The diagnosis of PD is based on clinical diagnostic criteria $[2,3]$ which carries 75 to $90 \%$ accuracy (based on clinicopathological correlation). Factors such as early presentation when signs of disease are subtle may reduce clinical certainty and delay diagnosis even in the hands of expert clinicians. Early neuropathological studies showed that more than $20 \%$ of subjects diagnosed with PD have an alternative diagnosis when compared with the pathologic gold standard [2]. More recent studies suggested that as many as $10 \%$ of PD patients in specialized movement disorders centers, and up to $25 \%$ of PD patients in general neurology offices, are misdiagnosed [3-5]. The cardinal motor features of parkinsonism are bradykinesia, rigidity, and tremor; among these, bradykinesia is considered the most sensitive [2]. The term 'clinically uncertain parkinsonian syndrome' (CUPS) has been used to describe cases with any of the following features: bradykinesia in the absence of other cardinal features of parkinsonism, rigidity and tremor without bradykinesia, atypical signs, signs of mild intensity, poor response to levodopa, and lack of disease progression [6]. By these criteria up to 1 in 5 patients with suspected PD will be classified as having CUPS [7]. In

\section{KARGER 125}

() 2015 S. Karger AG, Base

$1660-2854 / 15 / 0152-0081 \$ 39.50 / 0$

E-Mail karger@karger.com

www.karger.com/ndd
Danny Bega, MD

Department of Neurology, Northwestern University Feinberg School of Medicine 710 N Lake Shore Drive, Abbott Hall, 1112 Chicago, IL 60611 (USA)

E-Mail danny.bega@ northwestern.edu 
these patients, accurate identification of whether the cause of symptoms is presynaptic degeneration is important for interpreting disease course, therapy, and prognosis. This underscores the need for ancillary tests to support clinical judgment and improve diagnostic accuracy in PD.

In the last decade a number of radioligands have been developed that allow functional imaging of dopaminergic terminals $[8,9]$. The most commonly used ligands bind to the dopamine transporter (DAT), a protein located in the presynaptic membrane which serves as a major portal for dopamine uptake [8]. The literature on DATSPECT (dopamine transporter imaging with single-photon emission computed tomography) demonstrates a high degree of sensitivity and specificity in detecting abnormal signal uptake in the striatum in patients with $\mathrm{PD}$ [10], and loss of dopamine transporters have been shown to parallel the nigrostriatal deficit of $\mathrm{PD}$ at postmortem [11]. In 2011 the FDA approved the use of DaTscan ${ }^{\mathrm{TM}}$ (General Electric, Inc.) for the evaluation of cases of suspected presynaptic dopaminergic deficiency such as idiopathic $\mathrm{PD}$, and as an adjunct in differentiating $\mathrm{PD}$ tremor from essential tremor (ET). There is limited post-marketing data on the use of DAT-SPECT in specialty movement disorders clinics, and it is unclear to what extent the level of clinician expertise narrows or widens the range of what is considered 'clinically uncertain', and to what extent this impacts ordering practices. This study aimed to determine which features led to physician uncertainty and ordering of DAT-SPECT scans specifically in a specialty movement disorders clinic setting, and subsequently, to analyze the impact of these imaging results on diagnosis and management decisions.

\section{Methods}

This is a retrospective data analysis designed to investigate the use of DAT-SPECT in a specialty movement disorders center. The study was conducted at Northwestern University Parkinson's disease and movement disorders center (NU PDMDC) which is staffed by 4 specialty trained movement disorders neurologists, and in which approximately 2,000 patients are seen annually. The database of all DAT-SPECT images ordered by movement disorders specialists at the NU PDMDC between July 2011 and July 2013 was reviewed. The DAT-SPECT protocol for image acquisition is provided as appendix A. The study was approved by the institutional review board. Clinic notes in the electronic medical record of each patient were reviewed by a physician who was not involved in the patient care. Data was collected regarding pertinent history, exam findings, suspected diagnosis, management plan, and reason for ordering scans. Subjects were only excluded if imaging was obtained for clinical trial purposes or if the scan was ordered by a physician not specializing in movement disorders. Images were previously interpreted by a trained radiologist who had no interaction with the patients or knowledge of the study. Subsequent office visit notes for up to 2 follow-up visits (ranging from one week to one year post-scan) were reviewed to determine if the diagnosis or management plan changed in response to imaging.

The frequency of each indication for ordering a scan was calculated for all physicians as a group and also compared between physicians to assess for individual differences in ordering practices. Pre and post scan diagnoses and medications were compared to determine whether there were major differences in practice in response to the imaging information. Clinical predictors of abnormal scans were assessed.

\section{Results}

83 DAT-SPECT scans were ordered over a 2 years period. Table 1 shows the reasons for diagnostic uncertainty that led to ordering scans, with the most common being (1) ET vs. PD $(21.7 \%, \mathrm{n}=18)$ and (2) drug-induced parkinsonism vs. PD $(21.7 \%, \mathrm{n}=18)$. The table also demonstrates that the most frequent reason for questioning a suspected diagnosis of ET was an asymmetric resting tremor; in 3 out of 5 of these cases a normal scan supported the initial diagnosis. Among all cases where the scan showed abnormal uptake, the most common reason for questioning a diagnosis of PD was because signs of parkinsonism were too subtle $(\mathrm{n}=9)$, and the next most common reason was because of evidence of microvascular disease on brain imaging $(n=7)$. In 59\% ( $n=49)$ of cases, a change in clinical diagnosis or medication regimen occurred within one visit after the scan (fig. 1). Figure 1 shows that the strongest clinical impact was seen when the indication for the scan was to differentiate ET from PD in which $72.2 \%$ ( $\mathrm{n}=$ 13) had a change in diagnosis, management, or both. Table 2 shows the breakdown of management changes, with initiation of dopaminergic therapy for PD being the most frequent post-scan change overall $(n=16)$. Representative cases are provided in figure 2 . In addition, the strongest predictor of an abnormal scan was the combination of bradykinesia and rigidity (72\%).

Only one physician ordered scans for the indication of 'patient request'; other ordering indications were fairly evenly distributed among the four physicians. There was some variability (from $45-75 \%$ ) in the likelihood that a given physician would make a clinical change despite a scan confirming their initial clinical impression, but all physicians were willing to change their clinical impression $(100 \%$ of cases, $n=17)$ in the context of scan results that conflicted with their initial clinical suspicion. 
Table 1. DaTscan results by ordering indication

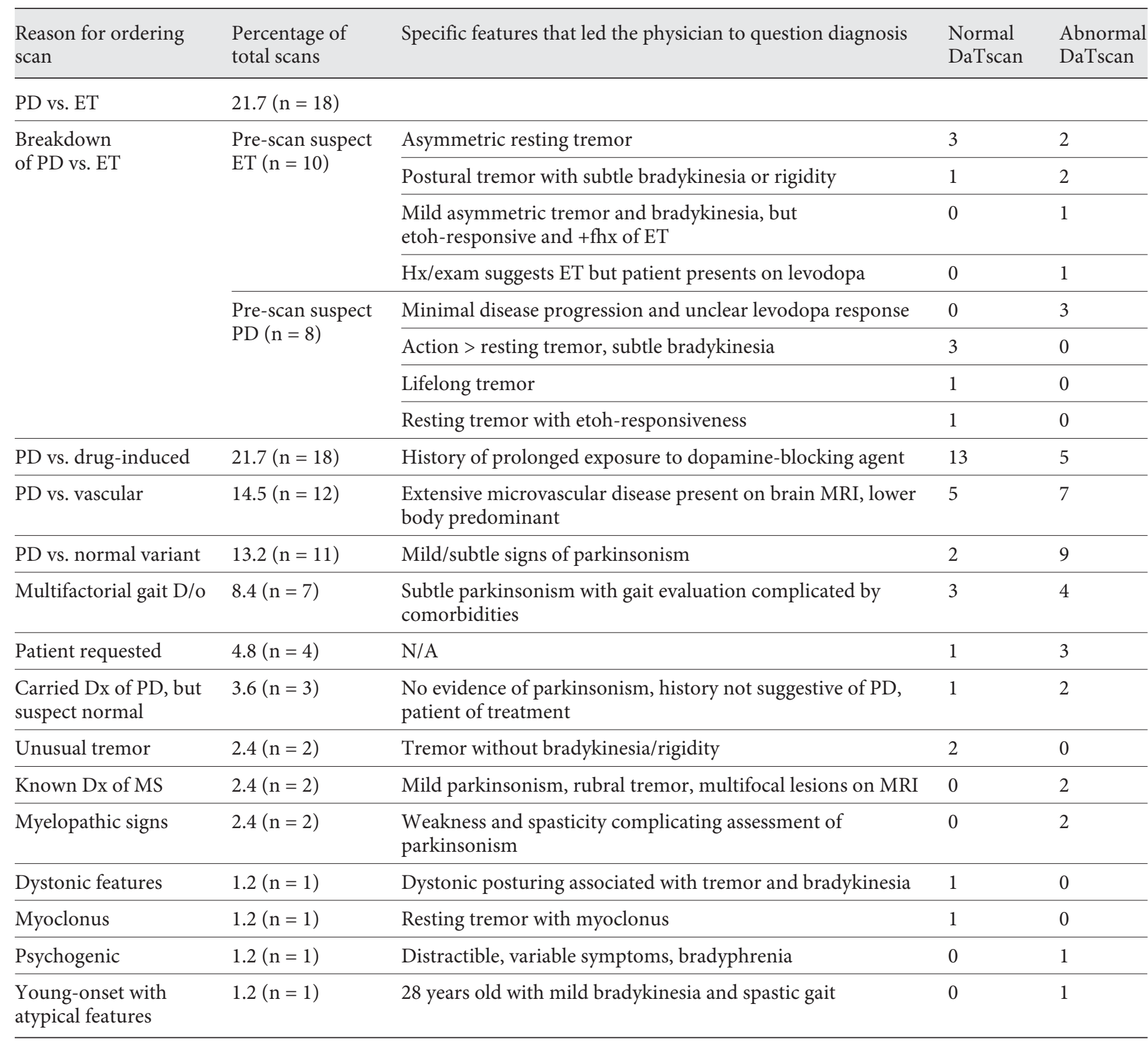

\section{Discussion}

The two most common indications for ordering DATSPECT scans in this specialized practice was to differentiate PD from ET and from drug-induced parkinsonism. As expected, no scans were ordered to differentiate PD from 'parkinson-plus' syndromes. The former practice (ET vs. PD) is consistent with the primary approved indication for DaTscan ${ }^{\mathrm{TM}}$ use, while the latter (PD vs. drug- induced parkinsonism) is not spelled out as a scan indication but is contained within the broader indication which is to establish the presence of presynaptic denervation. Previous studies have indicated that DAT-SPECT confirms presynaptic dopamine deficiency in clinically probable cases of PD about $90 \%$ of the time, and has good sensitivity and specificity (95 and $93 \%$, respectively) in discriminating PD from ET $[9,10]$. Our study shows that there is surprisingly high level of clinical uncertainty even 
Table 2. Change in management by ordering indication

\begin{tabular}{|c|c|c|c|c|}
\hline PD vs. ET & 4 & 0 & 1 & 2 \\
\hline PD vs. drug & 1 & 2 & 1 & 0 \\
\hline PD vs. vascular & 3 & 1 & 1 & 0 \\
\hline Early PD vs. normal variant & 2 & 2 & 1 & 0 \\
\hline
\end{tabular}

* Misc management changes in this category include initiation of medication for ET, referral for DBS, and initiation of medication for dystonia.

** Other includes psychogenic parkinsonism, dystonic tremor, lesional parkinsonism, neuropathy, etc. (see table 1 for all categories).

Fig. 1. Shows the number of scans ordered for each indication, which resulted in change in diagnosis, change in management, or no change respectively. In some instances, the same scan resulted in both a change in diagnosis and a change in management. * Refers to subtle findings of uncertain significance. ${ }^{* *}$ Refers to other possible diagnoses including psychogenic parkinsonism, dystonic tremor, multiple sclerosis related (lesional), neuropathy and myelopathy associated findings, and multifactorial gait disorders.

Fig. 2. DaTscan Images from Representative Cases. a A 66-year-old woman with schizoaffective disorder chronically treated with antipsychotic medications presented with bilateral resting tremor and had parkinsonism on exam. The initial diagnosis was 'unspecified parkinsonism', and the scan indication was idiopathic PD versus drug-induced parkinsonism. The scan was read as normal, and a clinical diagnosis of drug-induced parkinsonism was given. b An 85-year-old woman presented for evaluation had 10 years of progressive asymmetric action tremor with mild response to alcohol and beta-blockers. On exam there was asymmetric bradykinesia, but no resting tremor or rigidity. Prior trial of levodopa/carbidopa was reported as ineffective. The initial diagnosis was essential tremor, and the scan indication was idiopathic $P D$ versus essential tremor. The scan was read as having abnormal uptake in the basal ganglia bilaterally consistent with presynaptic dopamine deficiency. A clinical diagnosis of PD was given and levodopa/ carbidopa was reinitiated and titrated to a higher dose that was effective.
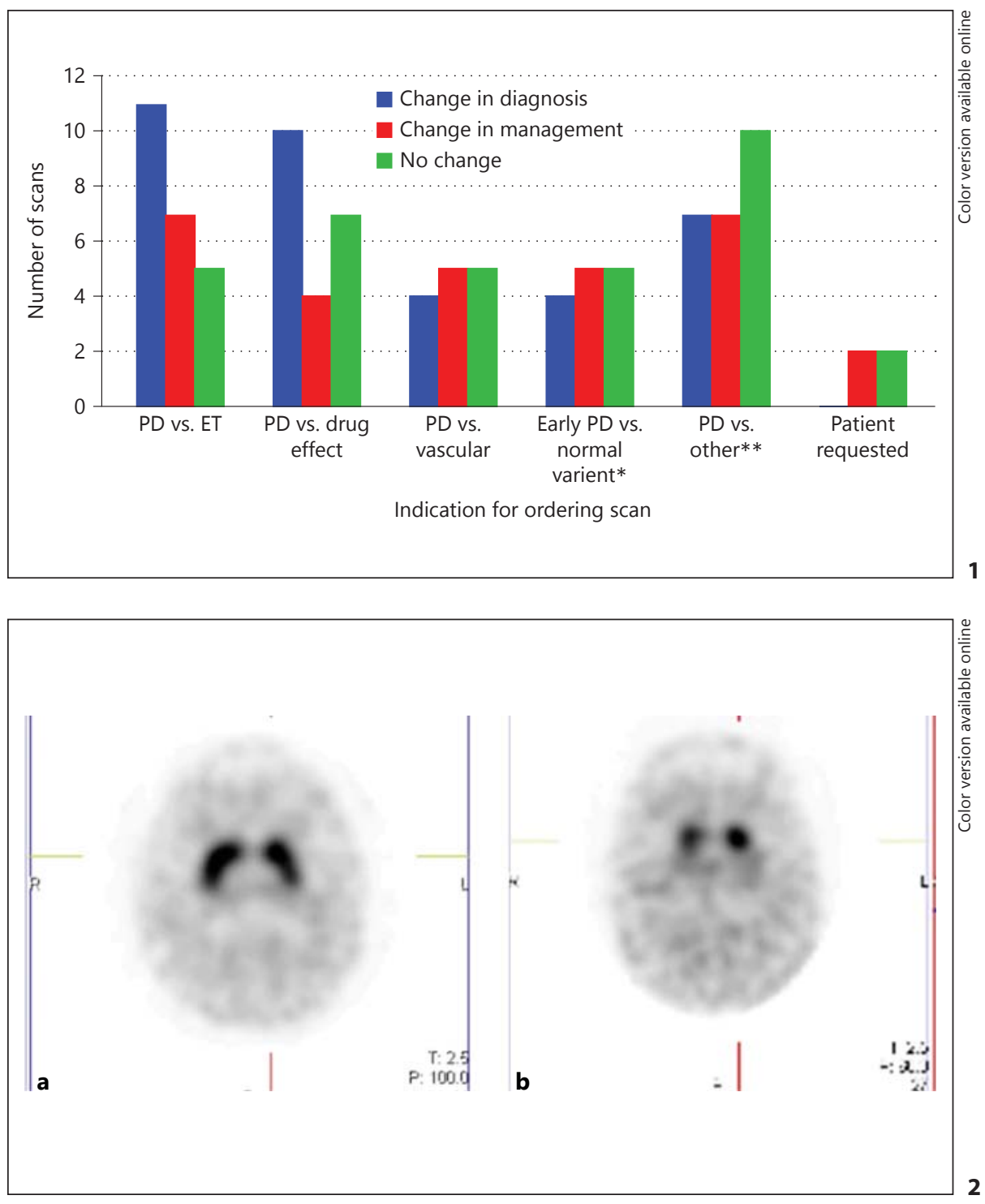
among expert clinicians to warrant the use of DATSPECT for differentiating PD from ET. Obviously this applies to a small proportion of patients with a non-classical tremor phenotype. In this study this included patients with suspected ET who had an asymmetric resting tremor, and patients with suspected PD who had a relatively static course, action greater than resting tremor, and/or absence of clear bradykinesia. The second most common indication for DaT-SPECT imaging was druginduced parkinsonism. Diagnostic uncertainty in these cases is not surprising as these patients present with a clinical phenotype indistinguishable from idiopathic PD, and only reversibility of parkinsonian features with time can clinically distinguish one from the other. This indication represents an important divergence from the preDAT 'wait and see' approach which has led to delay in establishing diagnosis and treatment especially in a time when the paradigm has shifted to earlier initiation of $\mathrm{PD}$ treatment.

Previous studies have indicated that DAT-SPECT leads to changes in clinical management in anywhere from $28-72 \%$ of patients $[6,12,13]$. In our study, a clinical impact of DAT-SPECT was seen in 59\% of all cases, with the strongest association seen for the indication of ET vs. PD (72\%). While the effect of scan results on patient confidence and acceptance of the diagnosis was not measured in this study, patient requested scans did result in medication initiation in 2 out of 4 patients who were previously reluctant to accept their diagnosis. Cost effectiveness was also not assessed, but a prior study suggested that the cost of imaging for the indication of PD versus ET may be relatively small compared to the financial and emotional gain associated with appropriate management [12].

Among the different management changes that were influenced by imaging in this study, initiation of dopaminergic therapy was the most frequent. Prior authors have suggested that DAT-SPECT scans are more likely to alter management when the results agree with the physician's initial clinical impression, and less likely to influence the decision of a specialist compared to a general neurologist $[6,7]$. These findings were not supported by our study. In fact, among the 17 cases that had clear physician pre-scan impressions that conflicted with final scan results, there was a post-scan change in diagnosis or management $100 \%$ of the time.

This study was limited in that it was a retrospective analysis at a single center which relied on interpretation of previously documented information. While changes in diagnosis and management were easily obtained from chart review and temporally correlated with DAT-SPECT imaging results, we cannot be certain that the changes were a direct result of the scan as opposed to other interval factors such as disease progression. Additionally, certain scan indications were by nature more likely than others to lead to management changes (i.e. in the case of PD vs. ET, either diagnosis could lead to medication initiation), while the clinical impact of other scan indications may have been underestimated by this measure.

In conclusion, diagnostic uncertainty in cases of parkinsonism exists even in a tertiary referral center, and DAT-SPECT is a tool that has significant impact on clinical diagnosis and management even in the hands of movement disorders specialists. This study provides data from a relatively large sample, including scans ordered by four experts, which will add to the growing literature on the post approval use of DAT-SPECT to help establish better practical guidelines for ancillary imaging in a specialty practice setting. Future studies will benefit from more accurate measures of the clinical impact and cost effectiveness of DAT-SPECT in these settings.

\section{Conflict of Interest}

Danny Bega, MD has no conflicts of interest to disclose. Paulina Gonzalez-Latapi, MD has no conflicts of interest to disclose. Cindy Zadikoff, MD has served as a consultant for UCB Pharma, Abbvie and Merz. She has also served as a speaker for Teva and GSK. William Spies, MD has no conflicts of interest to disclose. Tanya Simuni, MD has served as a consultant and received honorarium from Novartis, Ibsen, General Electric, UCB Pharma, TEVA, IMPAX, Merz, Boehringer Ingelheim, National Parkinson Foundation, and GSK (terminated April 2009). She has also received research support from NIH, MJ Fox Foundation, TEVA, IMPAX, National Parkinson Foundation, Northwestern Memorial Foundation.

\section{Appendix A}

\section{Image Acquisition}

Each patient received an oral dose of Lugol's iodine solution (10 drops in water) approximately one hour prior to radiopharmaceutical administration for thyroid blocking. In the event the patient had a history of allergy to iodine, $200 \mathrm{mg}$ potassium perchlorate given orally 30 minutes prior to radiopharmaceutical administration was used as an alternative. 3-5 mCi (111-185 MBq) of ${ }^{123} \mathrm{I}-$ Ioflupane were administered intravenously and imaging was performed between 3-6 hours after radiopharmaceutical administration. Imaging was performed on a dual head SPECT-CT hybrid camera with an integrated 16 slice CT scanner (Siemens Symbia). A low-energy, high-resolution parallel hole collimator was used. SPECT imaging was performed with 360 degree acquisition, using a circular orbit with a $14 \mathrm{~cm}$ radius, step and shoot acquisition at
Role of DAT-SPECT in Specialty

Movement Disorders Practice
Neurodegener Dis 2015;15:81-86 DOI: $10.1159 / 000370116$ 
15 seconds/view and 128 angular samples, 1.23 zoom. A $159 \mathrm{KeV}$ photopeak with a $10 \%$ window was used. A patient head holder was used, along with ${ }^{57} \mathrm{Co}$ markers for image registration. Image reconstruction was performed using Flash 3-D iterative reconstruction with a $128 \times 128$ matrix, post-processing using a $6 \mathrm{~mm}$ Gaussian filter ( 6 iterations, 8 subsets). A low-dose, non-contrast CT acquisition of the head was performed, using $130 \mathrm{KV}$, an effective 130
$\mathrm{mAs}$ and a $25 \mathrm{~s}$ scan time. CT reconstruction was performed with $5 \mathrm{~mm}$ slice thickness and $5 \mathrm{~mm}$ reconstruction. The SPECT and CT images were aligned to the same plane. The CT images were used for attenuation correction and image fusion. The reconstructed SPECT and SPECT-CT images were evaluated visually, and semiquantitative ROI analysis was also performed, with calculation of left:right and anterior:posterior striatal uptake ratios.

\section{References}

1 Kagi G, Bhatia K, Tolosa E: The role of DATSPECT in movement disorders. J Neurol Neurosurg Psychiatry 2010;81:5-12.

$\checkmark 2$ Hughes A, Daniel S, Kilford L, et al: Accuracy of clinical diagnosis of idiopathic Parkinson's disease: a clinic-pathological study of 100 cases. J Neurol Neurosurg Psychiatry 1992;55: 181-184.

3 Hughes A, Ben-Shlomo Y, Daniel S, et al: What features improve the accuracy of clinical diagnosis in Parkinson's disease: a clinicopathologic study. Neurology 2001;57:S34S38.

4 Felicio A, Godeiro-Junior C, Shih MC, et al: Evaluation of patients with clinically unclear parkinsonian syndromes submitted to brain SPECT imaging using the technetium-99m labeled tracer TRODAT-1. J Neurol Sci 2010; 291:64-68.
5 Meara J, Bhowmick B, Hobson P: Accuracy of diagnosis in patients with presumed Parkinson's disease. Age Aging 1999;28:99-102.

6 Catafau AM, Tolosa E: Impact of dopamine transporter SPECT using 123I-Ioflupane on diagnosis and management of patients with clinically uncertain parkinsonian syndromes. Mov Disord 2004;19:1175-1182.

7 Kupsch A, Bajaj N, Weiland F, et al: Impact of DaTscan SPECT imaging on clinical management, diagnosis, confidence of diagnosis, quality of life, health resource use and safety in patients with clinically uncertain parkinsonian syndromes: a prospective 1-year followup of an open-label controlled study. J Neurol Neurosurg Psychiatry 2012;83:620-628.

8 Marek KL, Seibyl JP, Zoghbi SS, et al: [123]I beta-CIT/SPECT imaging demonstrates bilateral loss of dopamine transporters in hemiParkinson's disease. Neurology 1996;46:231237.

-9 Parkinson Study Group: A multicenter assessment of dopamine transporter imaging with DOPASCAN/SPECT in parkinsonism. Neurology 2000;55:1540-1547.
0 Benamer HT, Oertel WH, Patterson J, et al: Prospective study of presynaptic dopaminergic imaging in patients with mild parkinsonism and tremor disorders: part 1. Baseline and 3-month observations. Mov Disord 2003;18: 977-984.

11 Niznik HB, Fogel EF, Fassos FF, et al: The dopamine transporter is absent in parkinsonian putamen and reduced in the caudate nucleus. J Neurochem 1991;56:192-198.

12 Van Laere K, Everaert L, Annemans L, et al: The cost effectiveness of 123I-FP-CIT SPECT imaging in patients with an uncertain clinical diagnosis of parkinsonism. Eur J Nucl Med Mol Imaging 2008;35:1367-1376.

13 Lokkegaard A, Werdelin LM, Friberg L: Clinical impact of diagnostic SPET investigations with a dopamine re-uptake ligand. Eur J Nucl Med Mol Imaging 2002;29:1623-1629. 